\title{
A study of the sintering behaviour of magnesium diboride
}

C. E. J. Dancer ${ }^{1 *}$, P. Mikheenko ${ }^{2}$, A. Bevan ${ }^{2}$, J. S. Abell ${ }^{2}$, R. I. Todd ${ }^{1}$, and C. R. M. Grovenor $^{1}$

${ }^{1}$ Department of Materials, University of Oxford, Parks Road, Oxford, OX1 3PH, UK.

${ }^{2}$ Department of Metallurgy and Materials, University of Birmingham, Edgbaston, Birmingham B15 2TT, UK.

* Corresponding author. Tel.: +44 1865 273766; fax: +44 1865273789 Email:

claire.dancer@linacre.oxon.org

\section{Abstract}

It is well known that a substantial increase in critical current density can be achieved by the heat-treatment of ex situ magnesium diboride powder-in-tube wires. However, it is not clear whether this is due to a true sintering process involving the significant transport of material and densification, or due for instance to the removal of volatile impurities from particle interfaces, limited chemical reactions at particle boundaries, or simply contact formation between particles by thermally activated direct adhesion. We believe that the term sintering in the magnesium diboride literature may often be used loosely when neither neck formation nor densification occurs during heat-treatment, and have designed experiments to understand what is happening during this processing step. We have studied the effect of a range of heat-treatments on the microstructure of pellets produced from commercial $\mathrm{MgB}_{2}$ powder using x-ray diffraction, scanning electron microscopy, Vickers hardness tests and by density measurements using Archimedes' principle. The results are compared to those from a dense sample produced from the same powder by resistive sintering. No significant densification is observed in pellets produced by conventional pressureless heat-treatment up to $1100{ }^{\circ} \mathrm{C}$. However, a clear 
correlation between density and hardness is established by comparison with results for bulk $\mathrm{MgB}_{2}$ produced by resistive sintering, which confirms that a classical sintering process has been induced in the latter samples.

\section{Keywords:}

$\mathrm{MgB}_{2}$, A. Firing, A. Sintering, B. Porosity, C. Superconductivity, D. Borides.

\section{Introduction}

Magnesium diboride $\left(\mathrm{MgB}_{2}\right)$ powder-in-tube (PIT) wires fabricated by the ex situ processing route (i.e. using pre-reacted $\mathrm{MgB}_{2}$ powder) have been studied extensively since the discovery of superconductivity in $\mathrm{MgB}_{2}$ in $2001^{1}$. In contrast to the more often studied in situ processing route, where the $\mathrm{MgB}_{2}$ phase forms during processing, ex situ processing does not result in a large density change since there is little reaction during heat-treatment ${ }^{2}$. Because the superconducting phase is present from the start, it is also possible to reduce impurity formation by limiting the temperature and duration of the heat-treatment stage. Ex situ $\mathrm{MgB}_{2}$ PIT tapes with critical current density $\left(\mathrm{J}_{\mathrm{c}}\right)$ of $10^{5}$ $\mathrm{A} / \mathrm{cm}^{2}$ (at $4.2 \mathrm{~K}, 0 \mathrm{~T}$ ) have even been produced with no heat-treatment. ${ }^{3}$ However, $\mathrm{MgB}_{2}$ PIT wires show substantially improved superconducting characteristics following heat-treatment at moderate temperatures. ${ }^{4}$ It has not been established whether this heattreatment is effective due to increasing the density of the $\mathrm{MgB}_{2}$ via conventional sintering processes, or whether some other mechanism such as removal of impurities or recrystallisation is occurring. ${ }^{5}$

Moderate heat-treatment temperatures of $900-1000{ }^{\circ} \mathrm{C}$ are typically used during $e x$ situ processing of PIT wires because at higher temperatures a thick reaction layer is formed between the sheath metal and the core. ${ }^{4,6} \mathrm{~A}$ wide range of grain sizes is 
generally evident in the cross-section of the wires. ${ }^{7}$ The main impurity phase formed is $\mathrm{MgO}^{8}$, although other phases such as $\mathrm{MgB}_{4}{ }^{9}$ and the non-stoichiometric phases $\mathrm{MgB}_{4+\delta}$ and $\mathrm{MgB}_{7+\delta}{ }^{10}$ have been identified in ex situ samples following heat-treatment. The effect of porosity in bulk ex situ $\mathrm{MgB}_{2}$ samples has been studied by Grinenko et al by systematically varying the density of bulk samples (with the same composition) between $16-70 \%$ of the maximum density (as calculated from the lattice parameters). ${ }^{11}$ As the sample density was decreased, the measured onset critical temperature $\left(T_{c, 0}\right)$ decreased, and the width of the superconducting transition increased significantly, indicating decreased connectivity between particles and decreasing the temperature range in which $\mathrm{MgB}_{2}$ can be used as a useful superconductor. In addition, denser material can be expected to reach higher transport critical currents than porous material processed by the same method, as the cross-sectional area of superconducting material is higher for a given sample diameter. ${ }^{12}$ We can thus conclude that it is important to understand how to control the density of the $\mathrm{MgB}_{2}$ in ex situ wires and tapes in order to achieve the optimum performance.

$\mathrm{MgB}_{2}$ powder can be heat-treated under pressure in vacuum or inert gaseous atmosphere to produce dense bulk $\mathrm{MgB}_{2}$, and heat-treatment at high gas pressure ${ }^{13}$, hot isostatic pressing (HIPing) ${ }^{14}$, and spark plasma sintering (SPS) ${ }^{15}$ have all been shown to be effective at improving bulk densities. Wires produced by HIPing have improved $\mathrm{J}_{\mathrm{c}}$ values in high magnetic fields compared with wires processed identically but heattreated conventionally at atmospheric pressure. ${ }^{16}$ Zhu et al studied TEM samples of bulk ex situ $\mathrm{MgB}_{2}$ heat-treated in high $\mathrm{Mg}$ vapour pressures, and their micrographs reveal a dense structure with some regions of fine-scale porosity, nano-sized $\mathrm{MgB}_{2}$ grains, and $\mathrm{MgO}$ impurities surrounding inclusions of large single-crystal $\mathrm{MgB}_{2}$ 
grains. ${ }^{13}$ There is clearly a significant difference in both microstructure and performance between $\mathrm{MgB}_{2}$ which has undergone high-pressure processing and that produced conventionally at atmospheric pressure. One of the aims of this work is to establish reliable metrics to measure these differences in microstructure.

The approach we have taken is to use the techniques developed for conventional structural ceramics and to apply them to bulk samples of $\mathrm{MgB}_{2}$. We designed experiments using bulk $\mathrm{MgB}_{2}$ to model what is happening in PIT wires during heattreatment, and as a result the maximum temperature was limited to $1100{ }^{\circ} \mathrm{C}$. These samples were compared to those produced using a non-conventional heat-treatment process which is known to produce dense samples, Resistive Sintering ${ }^{17}$. The starting powder was also comprehensively characterized to examine the impurities and the shape and size distributions of the particles.

\section{Experimental Details}

Commercial $\mathrm{MgB}_{2}$ powder (Alfa Aesar, $98 \%$ purity, -325 mesh) was used to produce all the bulk materials studied in this work. Scanning electron microscopy (SEM) in a JEOL 840F was performed at $5 \mathrm{kV}$ on powders dispersed on a carbon tab and coated with $2 \mathrm{~nm}$ of platinum to minimize charging. Transmission electron microscopy (TEM) using a JEOL 2000FX was carried out on $\mathrm{MgB}_{2}$ powder dispersed in isopropanol onto holey carbon films on a $\mathrm{Cu}$ grid. The particle size distribution was measured using a Malvern Mastersizer S Laser Diffraction system, using isopropanol as the dispersion medium. Laser diffraction was used in preference to microscopy to assess particle size because it can study a very wide range of particle sizes in one fast measurement. ${ }^{18}$ 
Bulk pellets ( $9 \mathrm{~mm}$ diameter, $\sim 4 \mathrm{~mm}$ height) were prepared from this powder by uniaxial pressing at 100 bar in a stainless steel die and heat-treatment for $1 \mathrm{~h}$ in flowing Ar gas, with peak temperatures ranging from $200-1100{ }^{\circ} \mathrm{C}$. The green pellets were placed in an $\mathrm{MgB}_{2}$ powder bed inside a closed alumina crucible to reduce $\mathrm{Mg}$ loss during heat-treatment. Density measurements were carried out on pellets before and after heat-treatment using the Archimedes method with isopropanol as the immersion liquid. Hardness values on the sample surfaces were measured by Vickers indentation. Pellets were fractured, and the resulting fracture surfaces were imaged by scanning electron microscopy again using the JEOL $840 \mathrm{~F}$ at $5 \mathrm{kV}$. To alleviate charging during imaging, samples were attached to aluminium stubs with silver paint and coated with 2 nm of platinum.

The increase in content of crystalline $\mathrm{MgO}$ following heat-treatment was calculated from area of XRD peaks by comparison to known mixtures of $\mathrm{MgO}$ and $\mathrm{MgB}_{2}$ content prepared from $\mathrm{MgO}$ powder (BDH Ltd) mixed with Alfa Aesar $\mathrm{MgB}_{2}$ powder. XRD scans were carried out on these calibration powder mixtures using a Philips $\theta-2 \theta$ diffractometer and $\mathrm{Cu}-\mathrm{K} \alpha$ radiation at $35 \mathrm{kV}$ and $50 \mathrm{~mA}$. The areas of the $\mathrm{MgB}_{2}$ (110) (at $2 \theta=59.8^{\circ}$ ) and $\mathrm{MgO}(220)\left(\right.$ at $2 \theta=62.3^{\circ}$ ) peaks were measured using the ProFit analysis program and their ratio calculated. The calibration curve (Fig. 1) generated was then used to measure any increase in $\mathrm{MgO}$ following heat-treatment in all subsequent samples. The detection limit below which the $(220)_{\mathrm{MgO}}$ peak becomes indistinguishable from the background noise was established as $\sim 5 \mathrm{wt} \%$.

A denser $\mathrm{MgB}_{2}$ sample was also produced from the same powder at the University of Birmingham using a resistive sintering apparatus (Fig. 2) which has previously been used to produce dense $\mathrm{MgB}_{2}$ bulk samples. ${ }^{17} \mathrm{MgB}_{2}$ powder was placed in the centre of 
a graphite die and pressed from both sides with tungsten plungers. Direct current was passed through the plungers, and is thought to lead to extreme local heating at the point contacts between particles. Typical pressure and temperature ranges measured of the graphite die are $42-55 \mathrm{MPa}$ and $900-980{ }^{\circ} \mathrm{C}$, but only represent average values across the whole sample. The optimum heat-treatment temperature was assessed by monitoring the thickness of the specimen during heat-treatment. The temperature was held at the level at which a rapid decrease in the height of the specimen occurred; i.e. at the temperature at which effective densification began. Once the height of the specimen stabilized, the sample was held at that temperature for a few minutes to ensure that the densification was complete.

\section{Results and Discussion}

\subsection{Characterization of commercial powder}

The particle size distribution measured by laser diffraction showed significant variation according to the dispersion protocol used (Fig. 3a). Initially, powder was added dry to the isopropanol used as a dispersion medium. Two further types of samples were also studied; (i) following stirring for various times (0-60 s) in isopropanol, and (ii) following ultrasonic dispersion for various times (0-600 s) in isopropanol. In order to easily compare the particle size distributions produced by the various dispersion methods, we determined the particle sizes for which $10 \mathrm{vol} \%$ of the particles were smaller than the given diameter $\left(\mathrm{d}_{10 \mathrm{vol} \%}\right)$, the median particle size $\left(\mathrm{d}_{50 \mathrm{vol} . \%}\right)$, and the particle size for which $90 \mathrm{vol} \%$ of the particles were smaller than the given diameter $\left(\mathrm{d}_{90 \mathrm{vol} . \%}\right)$. While the stirring time did not appear to have much effect on the particle size distribution, ultrasonic dispersion made a dramatic difference although it required 
considerable optimisation before the best results were obtained. Ultrasonic dispersion for $300 \mathrm{~s}$ produced the particle size distribution with greatest reduction in agglomerate size, as indicated in Fig. 4 (by measurement of the smallest $d_{10 v o l . \%}$ ). Increasing the duration of ultrasonic dispersion resulted in agglomeration, perhaps due to heating during the process, while shorter times did not break down the pre-existing agglomerates in the starting powder. In previous studies, Flükiger et al ${ }^{19}$ measured the particle size of commercial $\mathrm{MgB}_{2}$ powder prior to ball milling, but did not indicate the dispersion method used prior to particle sizing or the method used to obtain the particle size distribution data. Their data are reasonably similar to ours for dry, non-dispersed $\mathrm{MgB}_{2}$ powder for the size range they measured $\square(1 \mu \mathrm{m}<\mathrm{d}<600 \mu \mathrm{m})$.

The particle size distribution of $\mathrm{MgB}_{2}$ powder also from Alfa Aesar but of a different batch number was measured in the same way to check the variation between batches (Fig. 3b). There is clearly a very significant difference in the two powder batches, both in the as-delivered size distribution and that after ultrasonic dispersion. The ultrasonic dispersion was still effective (indicating considerable agglomeration in both powders) but this analysis indicates the importance of particle size distribution measurements to understand the extent of agglomeration in individual batches of $\mathrm{MgB}_{2}$ powder. Kováč et al have previously observed variations in performance of PIT wires produced from different batches of Alfa Aesar $\mathrm{MgB}_{2}{ }^{20}$ This variation was attributed to the differences in $\mathrm{MgO}$ content between the two batches, but our findings indicate that particle size and extent of agglomeration may also be a factor determining the final properties of the wires.

A combination of SEM and TEM was used to examine individual particles because of the wide size range involved. SEM images (Fig. 5a-c) confirm the size of 
large agglomerates as observed by laser diffraction, while additionally revealing the shape of the particles. Similarly TEM images (Fig. 5d-f) indicate that the finer material varies significantly in size ranging from agglomerates of up to $1 \mu \mathrm{m}$ in diameter to individual $50 \mathrm{~nm}$ diameter grains, similar to the range of particle sizes observed by laser diffraction. The wide size range is in agreement with SEM observations made by Kováč et al $^{20}$ who recorded particle/agglomerate sizes of $1<\mathrm{d}<60 \mu \mathrm{m}$. As indicated in the selected images, the shape of the particles and agglomerates varies considerably, which will affect the packing of these particles in the green compact prior to heat-treatment.

\subsection{Conventionally Heat-Treated Pellets}

All $\mathrm{MgB}_{2}$ green compacts had relative densities $65 \pm 3 \%$ of the maximum density, the latter being calculated from lattice parameters $\left(2.63 \mathrm{~g} / \mathrm{cm}^{3}\right)$. Following heattreatment at temperatures up to $1100{ }^{\circ} \mathrm{C}$, few samples showed any improvement in density, most values falling within the experimental error of $\pm 1 \%$ (Fig. 6). Overall these very modest density fluctuations can be attributed solely to the loss of volatile impurity content rather than to any true sintering. X-ray diffraction scans of heat-treated pellets indicate a steady increase in crystalline $\mathrm{MgO}$ content with increasing heattreatment temperature (Fig. 7). The increase in $\mathrm{MgO}$ content from the starting powder was calculated from peak area ratios and the calibration curve in Fig. 1 to be 5.3-7.0 \% for temperatures between 950 and $1100{ }^{\circ} \mathrm{C}$.

The density fluctuations observed cannot be directly correlated with the increase in $\mathrm{MgO}$ content which increases steadily with heat-treatment temperature. This may be due to the change in density caused by $\mathrm{MgB}_{4}$ formation during heat-treatment following evaporation of magnesium at high temperatures. Peaks in the XRD $\theta-2 \theta$ spectra attributable to $\mathrm{MgB}_{4}$ for heat-treatments at $1100{ }^{\circ} \mathrm{C}$ and $1050{ }^{\circ} \mathrm{C}$ have been labelled in 
Fig. 7. The $\mathrm{MgB}_{4}$ content has not been quantitatively investigated due to the lack of appropriate standards. It is clear however that the density fluctuations are significantly smaller than that required for any approach to full densification, which would require a density change in Fig. 6 of $\sim 35 \%$.

Hardness measurements can be directly correlated with the evolution of a sintered microstructure since increasing inter-particle connectivity leads to a significantly increased resistance to indentation. This relationship has previously been demonstrated by Mukhopadhyay et al, for instance, for silicon nitride and sialon. ${ }^{21}$ Fig. 8 shows the measured hardness of pellets as a function of increasing heat-treatment temperature. An increase in hardness from $35 \mathrm{HV}$ to $44 \mathrm{HV}$ is evident between the green compact (corresponding to heat-treatment temperature $25{ }^{\circ} \mathrm{C}$ in Fig. 8) and the sample heattreated at $200{ }^{\circ} \mathrm{C}$. This small increase is thought to be due to better connectivity as a result of evaporation of adsorbed volatile impurities. At higher temperatures the hardness remains between 40 and $50 \mathrm{HV}$ for heat-treatment temperatures up to $1000{ }^{\circ} \mathrm{C}$, above which a modest increase to 70 and then to $90 \mathrm{HV}$ at $1100{ }^{\circ} \mathrm{C}$ is observed. While these high temperature hardness values represent an increase from the green compact, the resulting hardness is still very much lower than that expected for fully dense $\mathrm{MgB}_{2}$. For comparison, Takano et al prepared highly dense samples using hot isostatic pressing and measured Vickers hardness values of 1700-2800 HV. ${ }^{22}$

The effects of heat-treatment on the hardness of pellets and on the cores of $\mathrm{MgB}_{2}$ PIT wires have been compared using the results above and similar measurements on wires produced by two-axis rolling in Bratislava, and also to a Fe-sheathed PIT wire produced by drawing in Oxford. The data for the Bratislava wires is taken from Hušek et $a l .{ }^{23}$ It is readily apparent that without heat-treatment the cores of all the wires are 
initially harder than the pellets (data plotted at $25{ }^{\circ} \mathrm{C}$ ). This is due to the extensive mechanical deformation which occurs during wire-drawing or two-axis rolling, leading to increased compaction of the core compared to uniaxial pressing of the pellets. No further increase in hardness occurs for the Oxford wire until heat-treatment above 800 ${ }^{\circ} \mathrm{C}$. For all three wires the hardness increases by approximately a factor of $1.5-3$ after heat-treatment, a similar ratio to that seen for the bulk pellets. However none of the wires achieved the high hardness values measured in hot isostatically-pressed samples ${ }^{22}$, indicating, as in the pellets, that there is no significant sintering behaviour. We believe that the modest increase in hardness is rather due to increased connectivity due to removal of volatile impurities, and formation of impurity phases between grains.

Careful observations of pellet fracture surfaces using SEM indicate that there is no change in appearance between green compact and heat-treated samples (Fig. 9a-c). The surfaces appear to be composed of separate particles, and significant charging occurs without careful preparation. There is no sign of the classical results of sintering, neck formation and occluded porosity. The porosity of the samples prevented polishing of sections due to severe particle pull-out.

\subsection{Resistively-Sintered Pellets}

Resistive sintering has a significantly greater effect on the density of bulk material than the conventional heat-treatments described above. This is not surprising given the very high local temperatures that are likely at point contacts between conducting particles, and also the high pressures applied. The resistively sintered sample produced from unmodified powder increased in density to $80 \%$ of the theoretical maximum density. The hardness of the sample was $1436 \mathrm{HV}$, which approaches that of hot isostatically pressed $\mathrm{MgB}_{2}{ }^{22}$, and clearly far exceeds the values of all our 
conventionally heat-treated $\mathrm{MgB}_{2}$ pellets and wires (Fig. 8). This must be due to a real increase in connectivity between particles, leading to increased resistance to indentation.

Evidence for significant sintering behaviour (including occluded porosity) was observed in SEM images of this sample. Parts of the sample had clearly undergone what could be described as classical sintering (Fig. 10), but adjacent areas indicated no change in particle connectivity and no neck formation (Fig. 10b), i.e. lacking the microstructural changes characteristic of sintering. Fig. 10c shows a region containing occluded porosity but where densification is not as complete as in Fig. 10a. This suggests that certain areas of the sample experienced much higher local temperatures and hence underwent more extensive sintering. Where current flow is not blocked by impurity particles, large pores and other defects, the high local heating induced by resistive sintering leads to greater densification. In other areas of the sample current flow is partially or fully blocked and hence the local heating does not occur. In these areas we presume that the local sample temperature is limited to the temperature of the die, which is below the temperatures used in the conventional heat-treatment experiments in this study. Alternatively, the differences in densification between adjacent areas may be due to particle packing differences in the green compact.

X-ray diffraction of the phases present in this sample gave similar results to the $\mathrm{MgB}_{2}$ sample conventionally heat-treated at $1100{ }^{\circ} \mathrm{C}$. The crystalline $\mathrm{MgO}$ content increased to $7.8 \%$, and peaks attributable to $\mathrm{MgB}_{4}$ were also observed. This is similar to the XRD results for the sample produced by pressureless sintering at $1100{ }^{\circ} \mathrm{C}$.

These observations suggest that resistive sintering can encourage very significant local sintering, and also demonstrates that conventional ceramic characterization techniques can be useful in studying sintering behaviour in $\mathrm{MgB}_{2}$ samples. 


\section{Conclusions}

We believe that we have conclusively demonstrated that after conventional ex situ heattreatments $\mathrm{MgB}_{2}$ powder shows very little sign of sintering beyond particle-particle adhesion even at $1100{ }^{\circ} \mathrm{C}$, well above the temperature limit for practical wire fabrication set by core/sheath interactions ${ }^{24}$. Two kinds of $\mathrm{MgB}_{2}$ wires show an increase in core hardness as a result of improved compaction during mechanical processing, but similarly limited evidence of significant sintering during pressureless heat-treatment. This suggests the very real probability that the promising superconducting properties already achieved in PIT wires ${ }^{7}$ may be significantly improved if sintering and densification can be encouraged. Conversely, pellets made by resistive sintering under modest applied pressures do display partially sintered microstructures, and a corresponding large increase in both density and hardness is observed. Ex situ resistively sintered bulk samples, where we do observe significant densification, have previously attained $\mathrm{J}_{\mathrm{c}, \mathrm{mag}}>10^{5} \mathrm{~A} / \mathrm{cm}^{2}$ in low fields at $20 \mathrm{~K}^{25}$, so we are optimistic that similar performance can be achieved in wires and tapes.

Another interesting observation is that characterization of the starting powder revealed severe agglomeration which will act as a significant source of porosity in artefacts produced from this powder unless the agglomerates can be broken down during processing, and we have suggested one possible way of achieving this. The particle size distribution of different batches of Alfa Aesar $\mathrm{MgB}_{2}$ powder also varies very significantly, but agglomeration was observed in both batches used in this study. 


\section{Acknowledgements}

CEJD acknowledges funding from the EPSRC through the University of Oxford

Department of Materials 2004 Doctoral Training Grant. The work at Birmingham was funded by an EU FP6 STREP grant HIPERMAG. We are grateful to the EPSRC Engineering Instrument Pool for the loan of the Malvern Mastersizer S laser diffraction system. The authors would also like to thank Dr Susannah Speller for her careful reading of the manuscript.

\section{References}

1. Nagamatsu, J., Nakagawa, N., Muranaka, T., Zenitani, Y. \& Akimitsu, J., Superconductivity at $39 \mathrm{~K}$ in magnesium diboride, Nature, 2001, 410, pp.63-4.

2. Zhou, S., Pan, A. V., Liu, H. \& Dou, S., Single-and multi-filamentary Fe-sheathed $M g B_{2}$ wires, Phys. C, 2002, 382, pp.349-54.

3. Grasso, G., Malagoli, A., Ferdeghini, C., Roncallo, S., Braccini, V., Siri, A. S. \& Cimberle, M. R., Large transport critical currents in unsintered $\mathrm{MgB}_{2}$ superconducting tapes, Appl. Phys. Lett., 2001, 79, pp.230-2.

4. Grasso, G., Malagoli, A., Modica, M., Tumino, A., Ferdeghini, C., Siri, A. S., Vignola, C., Martini, L., Previtali, V. \& Volpini, G., Fabrication and properties of monofilamentary $\mathrm{MgB}_{2}$ superconducting tapes, Supercond. Sci. Technol., 16, pp.271-5.

5. Fabbricatore, P., Greco, M., Musenich, R., Kováč, P., Hušek, I. \& Gomory, F., Influence of the sintering process on critical currents, irreversibility lines and pinning energies in multifilamentary $\mathrm{MgB}_{2}$ wires, 2003, Supercond. Sci. Technol., 16 pp.364-70.

6. Kováč, P., Hušek, I., \& Melišek, T., Transport currents of two-axially rolled and post-annealed $\mathrm{MgB}_{2} / \mathrm{Fe}$ wires at $4.2 \mathrm{~K}$, Supercond. Sci. Technol. 2002, 15, pp.13404.

7. Kováč, P., Hušek, I., Grovenor, C. \& Salter, C. Properties of as-deformed and postannealed MgB2/Fe(Fe-alloy) composite wires, Supercond. Sci. Technol., 2003, 16, pp.292-6.

8. Kumakura, H., Matsumoto, A., Fujii, H. \& Togano, K., High transport critical current density obtained for powder-in-tube-processed $M g B_{2}$ tapes and wires using stainless steel and Cu-Ni tubes, Appl. Phys. Lett., 2001, 79, pp.2435-7.

9. Lezza, P., Gladyshevskii, R., Suo, H. L. \& Flukiger, R. F. Quantitative study of the inhomogeneous distribution of phases in Fe-sheathed ex situ $\mathrm{MgB}_{2}$ tapes, Supercond. Sci. Technol. 2005, 18, pp.753-7.

10. Eyidi, D., Eibl, O., Wenzel, T., Nickel, K. G., Schlachter, S. I. \& Goldacker, W., Superconducting properties, microstructure and checmical composition of $\mathrm{MgB}_{2}$ sheathed materials, Supercond. Sci. Technol., 2003, 16, pp.778-88.

11. Grinenko, V., Krasnoperov, E. P., Stoliarov, V. A., Bush, A. A. \& Mikhajlov, B. P., Superconductivity in porous $\mathrm{MgB}_{2}$, Solid State Comm., 2006, 138, pp.461-5. 
12. Pan, A.V., Zhou, S., Liu, H. \& Dou, S., Properties of superconducting $M g B_{2}$ wires: in situ versus ex situ reaction technique, Supercond. Sci. Technol., 2003, 16, pp.639-44.

13. Zhu, Y., Wu, L., Volkov, V., Li, Q., Gu, G., Moodenbaugh, A. R., Malac, M., Suenaga, M. \& Tranquada, J., Microstructure and structural defects in $\mathrm{MgB}_{2}$ superconductor, Physica C, 2001, 356, pp.239-53.

14. Maple, M.B., Taylor, B. J., Frederick, N. A., Li, S., Nesterenko, V. F., Indrakanti, S. S. \& Maley, M.P., Critical scaling and flux dynamics in bulk $\mathrm{MgB}_{2}$ and high-purity $\mathrm{YBa}_{2} \mathrm{Cu}_{3} \mathrm{O}_{7-\delta}$, Phys. C, 2002, 382, pp.132-6.

15. Lee, S.-Y., Yoo, S.-I., Kim, Y.-W., Hwang, N. M. \& Kim, D.-Y., Preparation of dense $\mathrm{MgB} \mathrm{B}_{2}$ bulk superconductors by spark plasma sintering, J. Am. Ceram. Soc., 2003, 86, pp.1800-2.

16. Serquis, A., Civale, L., Hammon, D. L., Liao, X. Z., Coulter, J. Y., Zhu, Y. T., Jaime, M., Peterson, D. E., Mueller, F. M., Nesterenko, V. F. \& Gu, Y., Hot isostatic pressing of powder in tube $\mathrm{MgB}_{2}$ wires, Appl. Phys. Lett., 2003, 82, pp.2847-9.

17. Mikheenko, P., Bevan, A. I. \& Abell, J.S., Nucleation and growth of dense phase in compressed $\mathrm{MgB}_{2}$, J. Phys.: Conference Series, 2006, 43, pp.535-8.

18. Reed, J. S., Principles of Ceramic Processing, John Wiley and Sons, New York, 1995.

19. Flükiger, R., Lezza, P., Beneduce, C., Musolino, N. \& Suo, H. L., Improved transport critical current and irreversibility fields in mono- and multifilamentary $\mathrm{Fe} / \mathrm{MgB}_{2}$ tapes and wires using fine powders, Supercond. Sci. Technol., 2003, 16, pp.264-70.

20. Kováč, P., Hušek, I., Melišek, T., Grivel, J. C., Pachla, W., Štrbík, V., Diduszko, R., Homeyer, J. \& Andersen, N. H., The role of $\mathrm{MgO}$ content in ex situ $\mathrm{MgB}_{2}$ wires, Supercond. Sci. Technol., 2004, 17, pp.L41-6.

21. Mukhopadhyay, A. K., Datta, S. K. \& Chakraborty, D., Hardness of silicon nitride and sialon, Ceram. Int., 1991, 17, pp.121-7.

22. Takano, Y., Takeya, H., Fujii, H., Kumakura, H., Hatano, T., Togano, K., Kito, H., \& Ihara, H., Superconducting properties of $M g B_{2}$ bulk materials prepared by highpressure sintering, Appl. Phys. Lett., 2001, 78, pp.2914-6.

23. Hušek, I., Kováč, P., Grovenor, C. R. M. \& Goodsir, L., Microhardness as a tool for the filament density and metal sheath analysis in $\mathrm{MgB} / \mathrm{Fe} /(\mathrm{Cu})$ wires, Supercond. Sci. Technol., 2004, 17, pp.971-6.

24. Flukiger, R., Suo, H. L., Musolino, N., Beneduce, C., Toulemonde, P. and Lezza, P., Superconducting properties of $\mathrm{MgB}_{2}$ tapes and wires Physica C, 2003, 385, pp.286305.

25. Mikheenko, P., Martinez, E., Bevan, A. I., Abell, J. S. \& MacManus-Driscoll, J. L. Grain boundaries and pinning in bulk $\mathrm{MgB}_{2}$, Supercond. Sci. Technol., 2007, 20, pp.S264-70. 


\section{Figures}

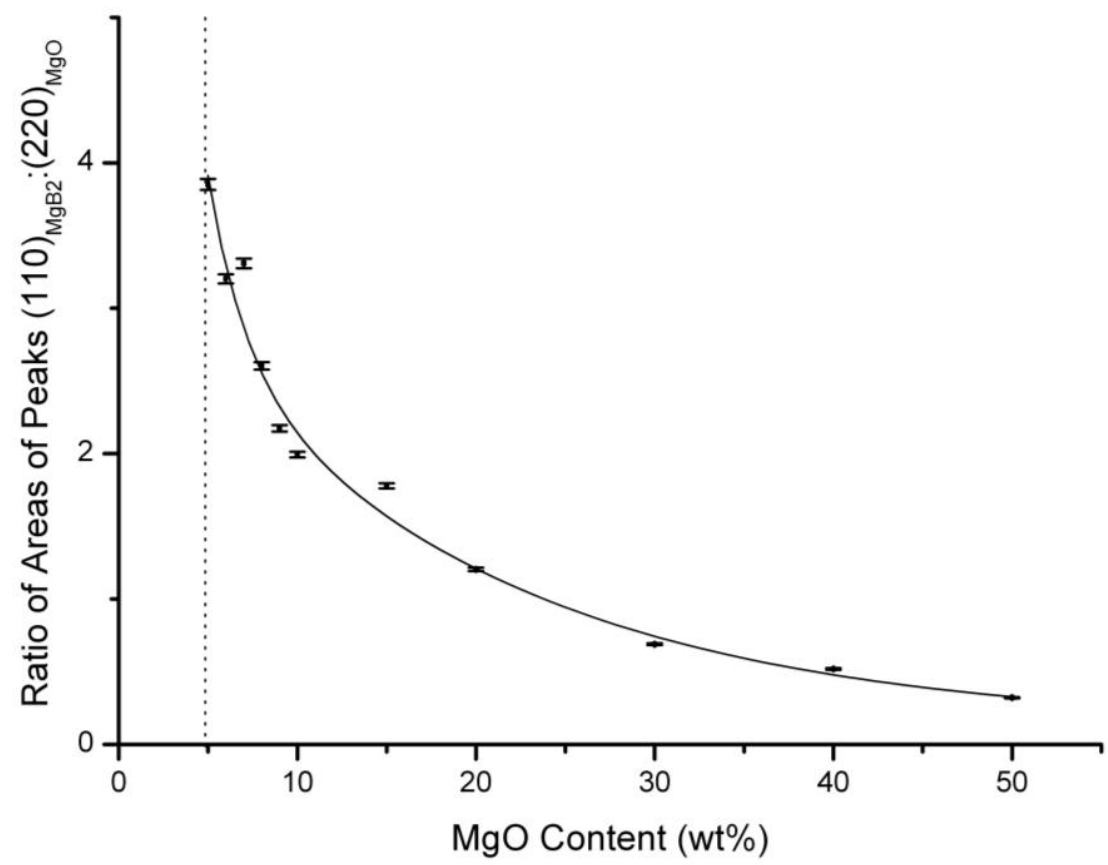

Fig. 1. MgO content XRD calibration curve.

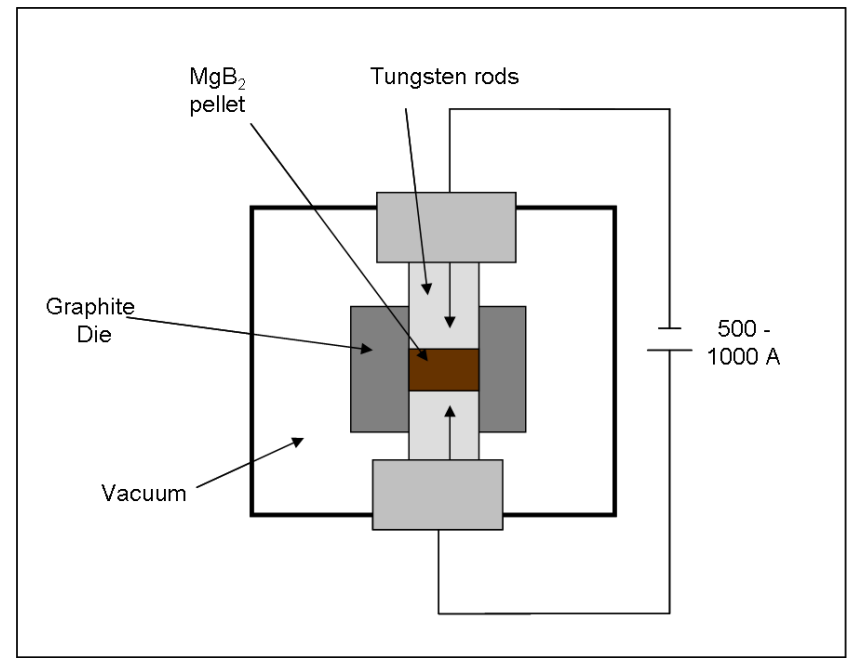

Fig. 2. Schematic diagram of resistive sintering apparatus. 

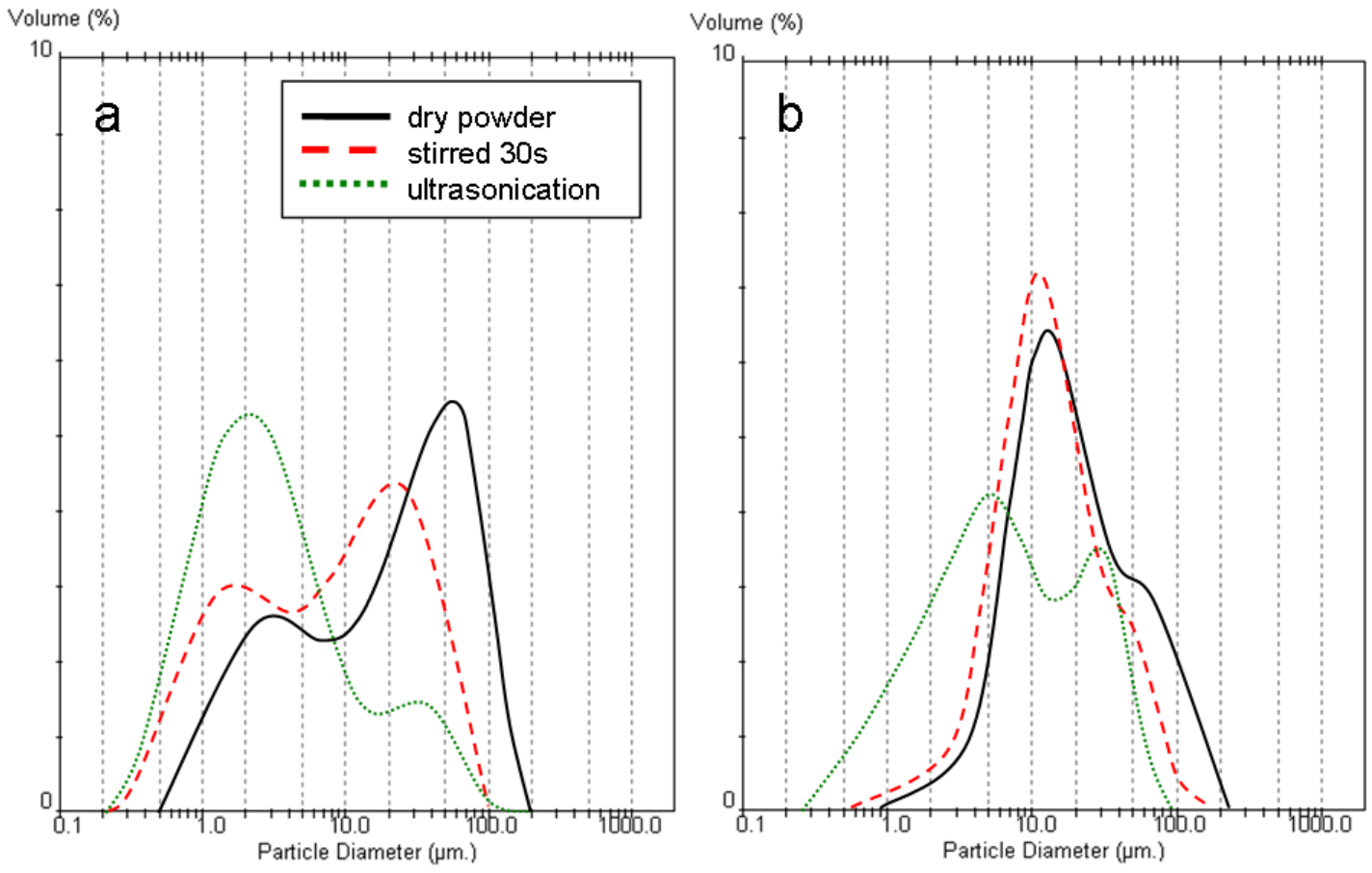

Figure 3 Laser diffraction particle size distribution for Alfa Aesar $\mathrm{MgB}_{2}$ powder a) Batch 1 (main batch used for bulk studies) b) Batch 2 .

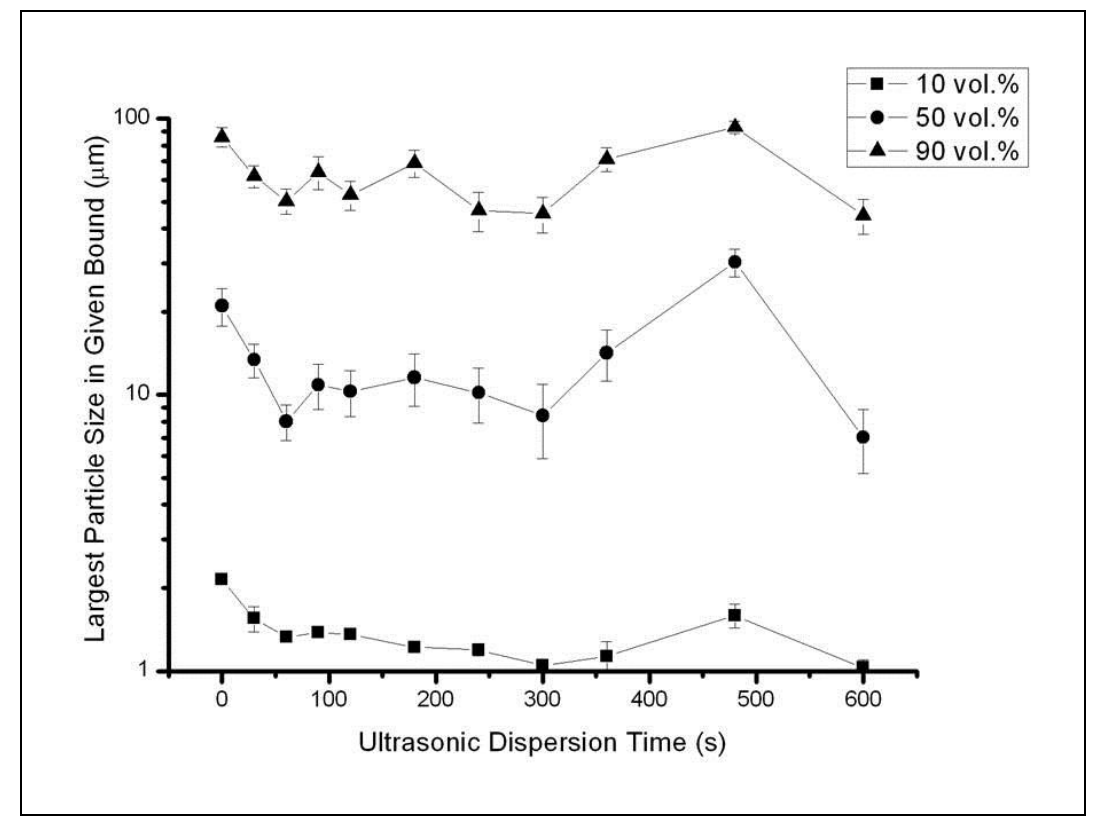

Figure 4 Variation of particle size with length of ultrasonic dispersion time. The error bars were calculated from the standard deviation of three separate measurements carried out on identical powder samples. 

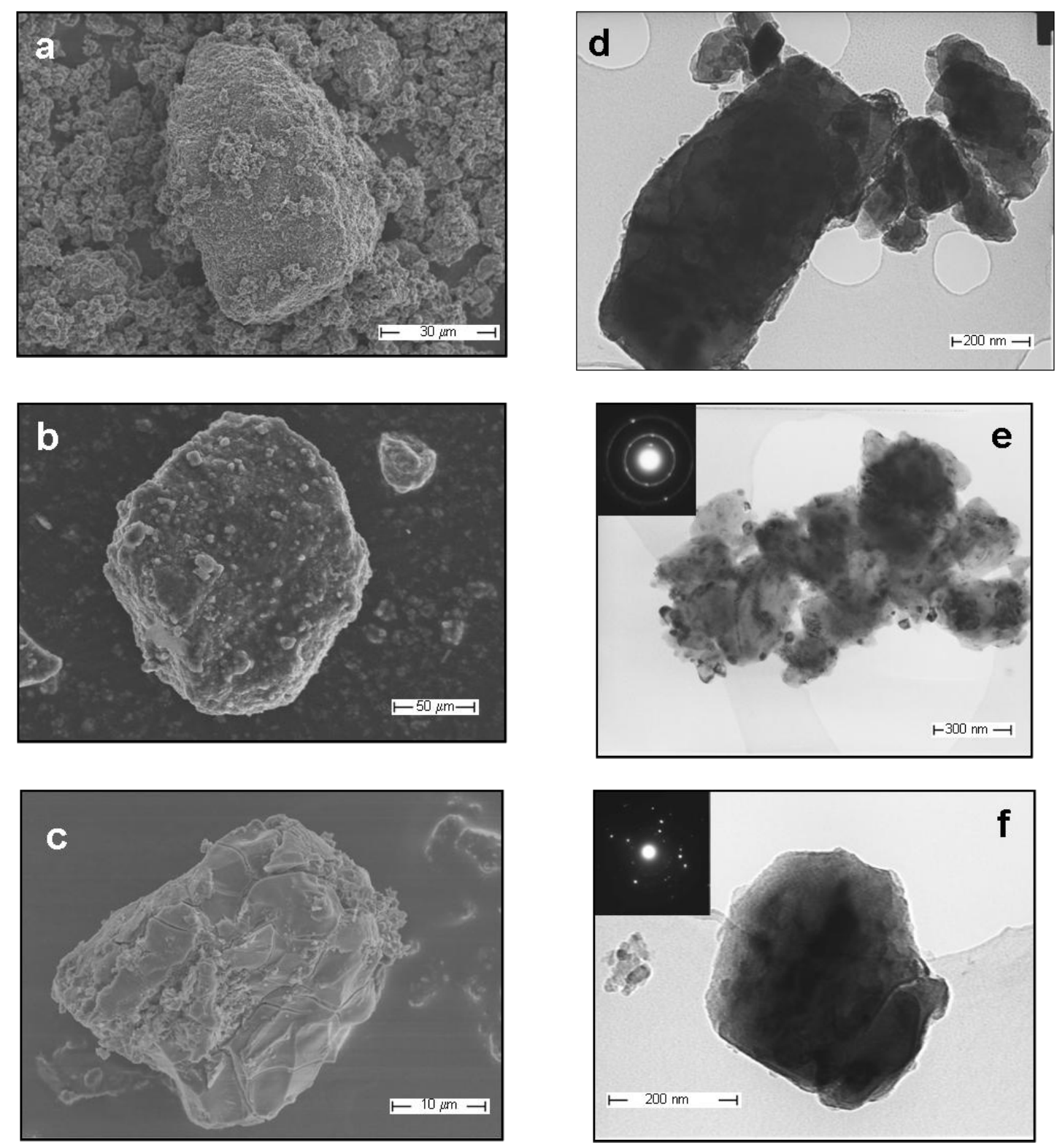

Figure 5 SEM (a-c) and TEM (d-f) images of typical particles and agglomerates found in Alfa Aesar $\mathrm{MgB}_{2}$ powder. 


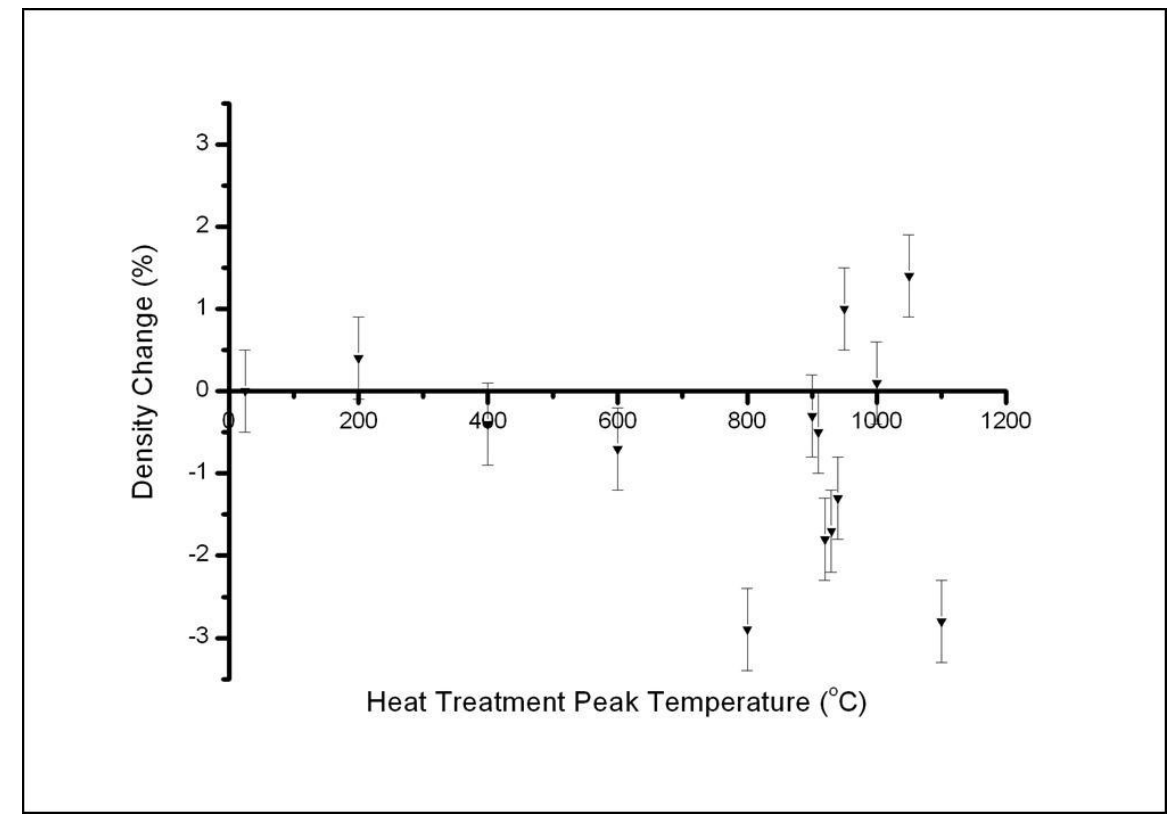

Figure 6 Change in density of $\mathrm{MgB}_{2}$ pellets after pressureless heat-treatment. 

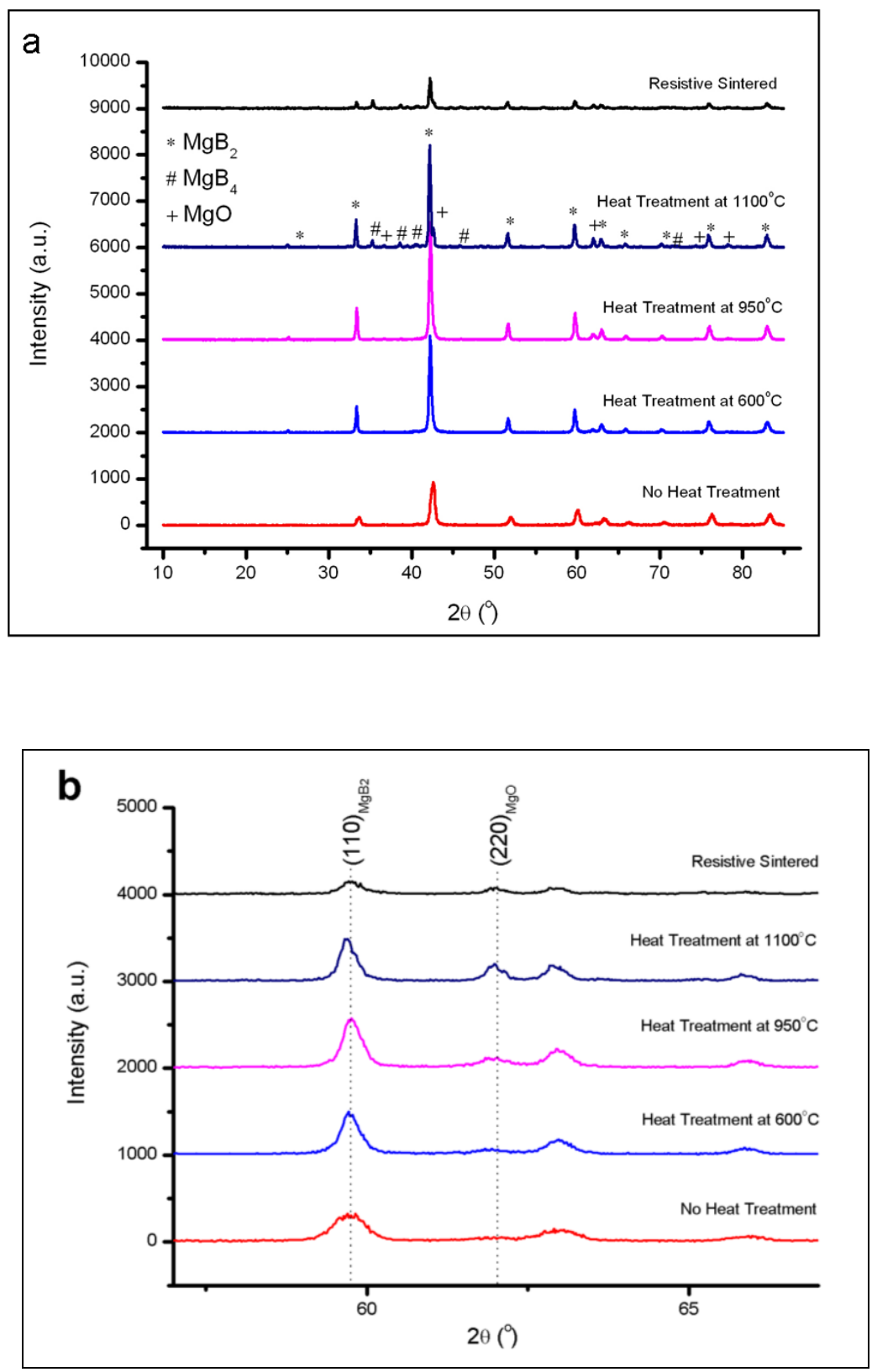

Figure $7 \mathrm{XRD}$ spectra of $\mathrm{MgB}_{2}$ powder and bulk pellets a) large angular range b) peaks used for $\mathrm{MgO}$ calibration measurements. The reduced intensity of the scans for the resistive sintered sample compared to the pressure-less heat-treated samples is due to the lower volume of the resistive sintered sample. 


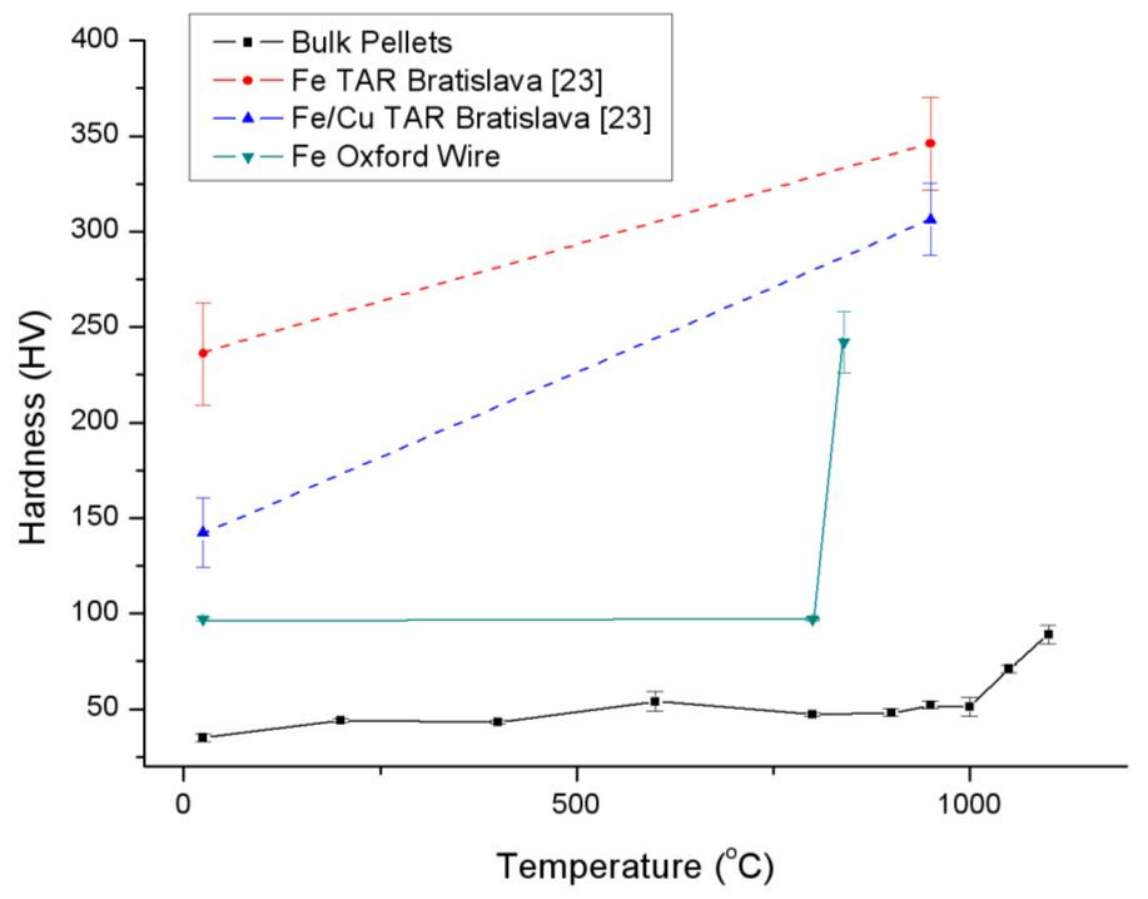

Figure 8 Hardness vs heat-treatment temperature for $\mathrm{MgB}_{2}$ pellets and wires fabricated from Alfa Aesar $\mathrm{MgB}_{2}$ powder. Note that the dashed lines are provided only as a guide to the eye, and are not intended to suggest a linear relationship (only limited hardness/temperature data was available for these samples). 

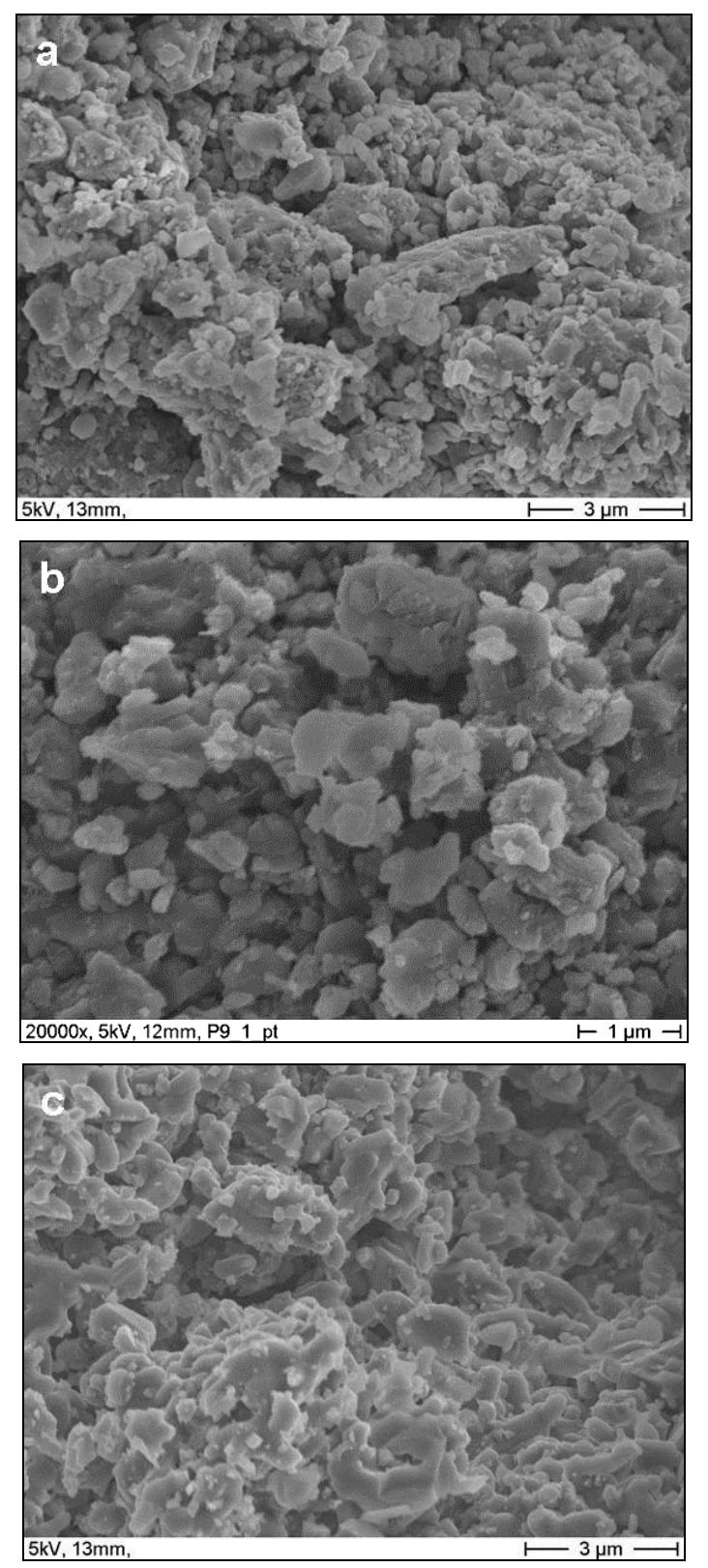

Figure 9 SEM images of fracture surfaces of $\mathrm{MgB}_{2}$ pellets

a) as-pressed (no heat-treatment)

b) pressure-less heat-treatment at $950^{\circ} \mathrm{C}$

c) pressure-less heat-treatment at $1100^{\circ} \mathrm{C}$. 


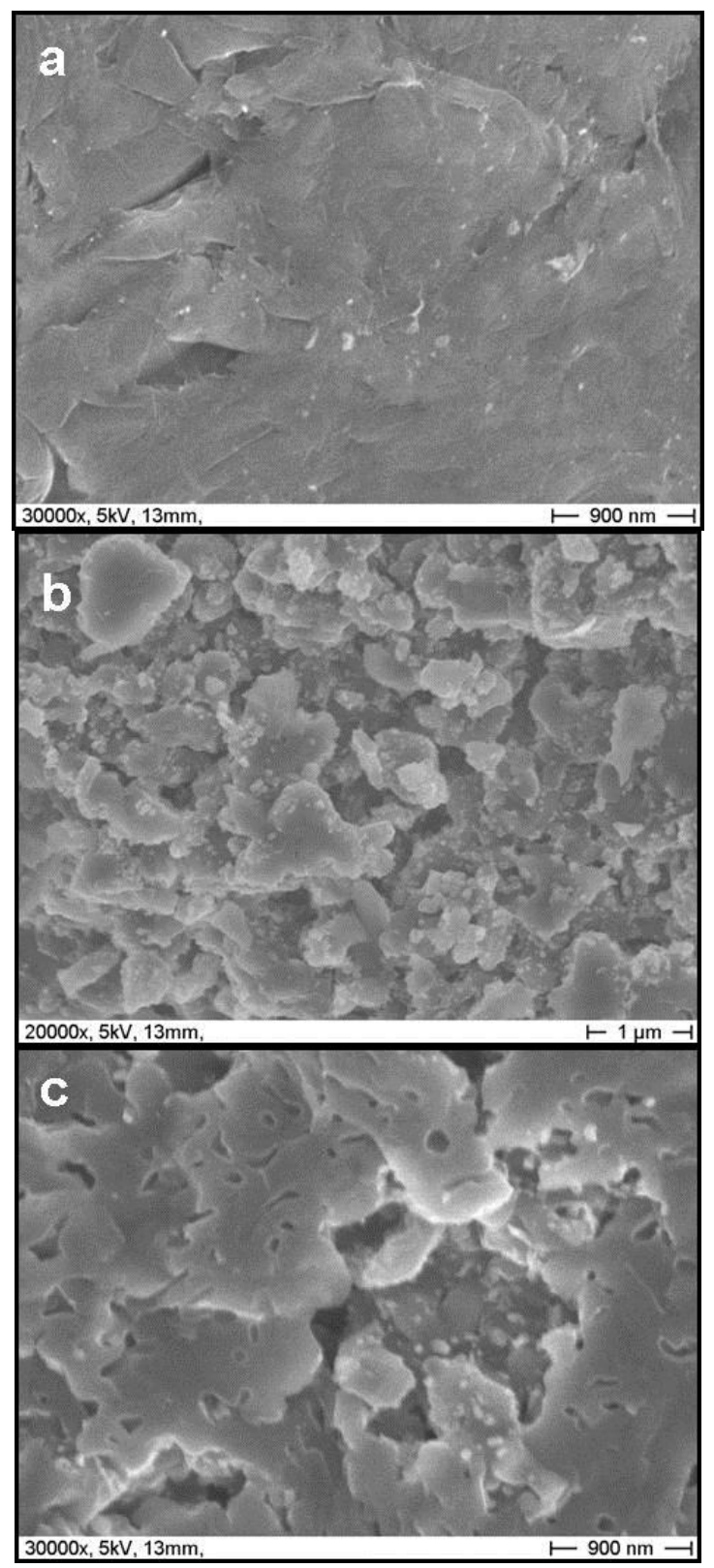

Figure 10 SEM images of fracture surfaces of resistive sintered $\mathrm{MgB}_{2}$ bulk a) Dense area

b) Porous area

c) Occluded porosity 